\title{
Persistent Interatrial Foramen Primum
}

\author{
BRADLEY M. PATTEN \\ Department of Anatomy, University of Michigan Medical School, \\ Ann Arbor, Michigan
}

Congenital interatrial septal defects close to the atrioventricular orifices are commonly regarded as the result of inadequate growth of interatrial septum primum. Unquestionably this is an important factor in their genesis but other critical related conditions are neglected in this oversimplified interpretation. The objective of this paper is to bring out these associated processes and to attempt to fit them into their proper perspective in the total picture, both as to structural features and as to the timing of their growth in relation to one another.

Perhaps the most effective way of approaching the subject is to scrutinize first the precise location and relations of these defects as seen postnatally, and to note, also, the range of their extensiveness. Al- though defects in this location are by no means as common as defects at the foramen ovale they have been well known over too many years to make it profitable to review in detail the voluminous literature pertaining to them. Suffice it to note that that the classical descriptions of Peacock (1866) and Rokitanski (1875) were followed by a plethora of case reports, and that the systematic survey of Maude Abbott ('32) and the recent critical studies of Edwards and Rogers ('48) and Edwards ('60) have brought abundant material together to give us adequate data on this defect as seen postnatally. It should be sufficient, therefore, to consider here a few cases selected to show the varying extent of these defects, and so oriented for illustration that the anatomical rela-

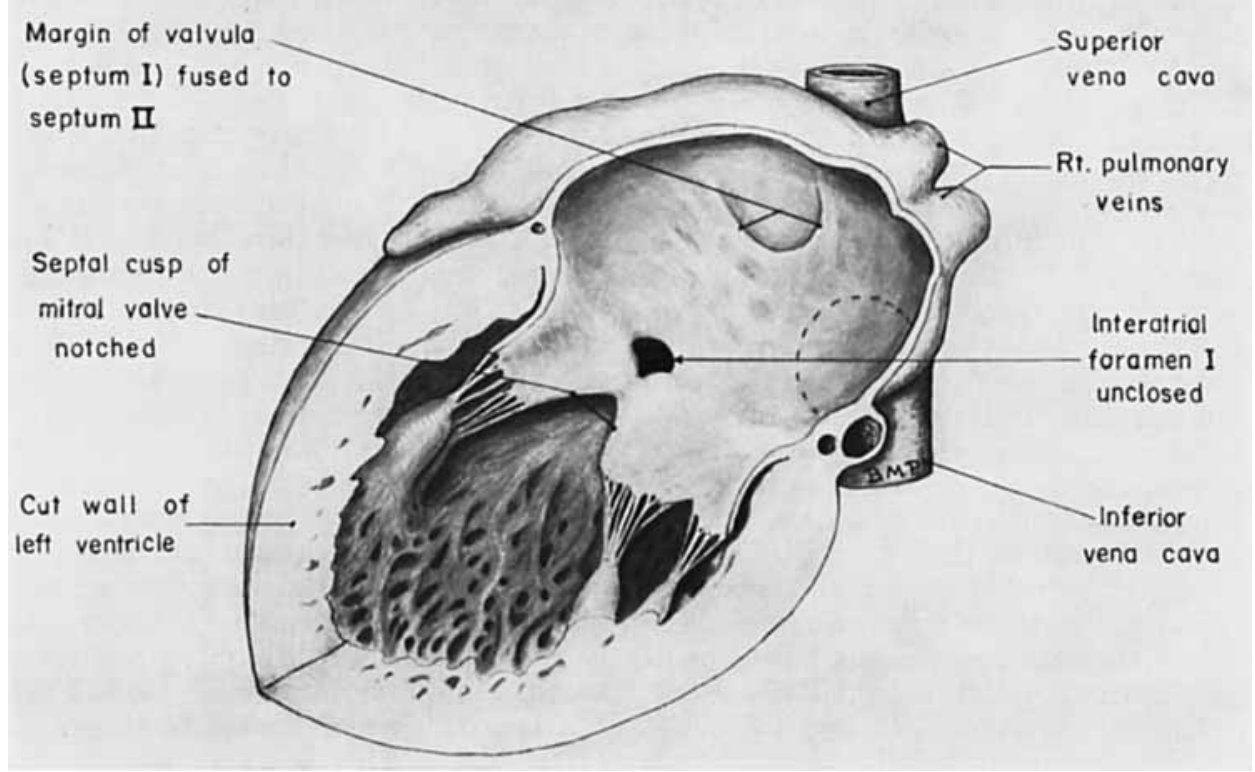

Fig. 1 Persistent interatrial foramen primum of minimal size. (From heart of a 60 year-old woman, Path.-anat. Inst., Vienna.) 
tion of the defect to other cardiac structures is clearly evident.

All the hearts illustrated as type cases were ones I had the opportunity of studying from museum or autopsy specimens when I was a visiting investigator at the Pathologisch-anatomisches Institut in Vienna. The case illustrated in figure 1 was selected as representing a persistent interatrial foramen of minimal extent but in a typical location and of characteristic shape. There is definite notching of the septal cusp of the mitral valve on its ventricular margin. On the attached base of the valve there is a definite groove, but below this groove the valve is continuous. This is a condition far less usual than complete separation of the atrioventricular valves as shown in figures 2 and 3. It may, I believe, be regarded as part of the picture to be expected when the defect is minimal in extent.

The heart shown in figures 2 and 3 was selected as representing an extensiveness near the middle of the range of defects of this type. It shows the involvement of the atrioventricular valves which is to be expected whenever defects in this location are other than minimal. The septal leaflet of the mitral, and the septal leaflet of the tricuspid valve are both completely divided (fig. 3 ) instead of being merely notched as was the case in the heart illustrated in figure 1.

The heart illustrated in figure 4 was chosen as an example of a defect in this location which was in the maximal size range. The unclosed foramen primum measured $57 \times 37 \mathrm{~mm}$. The septal leaflets of both atrioventricular valves were completely divided exposing the margin of the muscular portion of the interventricular septum. It is important in making surgical closure of such defects to realize that the main atrioventricular bundle lies just beneath the endocardium of this exposed part of the septum.

The clinical histories on these cases were meager but there were enough notes to indicate that the individuals had shown the characteristic conditions described for this defect by Taussig ('47) and Edwards ('60). These may be briefly summarized as follows. With the full functioning of the pulmonary circuit attained postna- tally, the flow in the presence of an interatrial defect is reversed from the fetal direction and becomes from left to right. This means that with each cardiac cycle the right side of the heart has an extra volume of blood to pump. The result is a progressively increasing dilation and hypertrophy of the right side of the heart, especially the right ventricle. The extra volume of blood going to the right ventricle results in an increased pulmonary flow with a resultant increase in transudation of plasma within the lungs. This is a situation which the pathologist frequently characterizes as a "wet lung." Apparently, it increases vulnerability to pulmonary infections. When such a shunt remains uncorrected the walls of the small arterial vessels of the pulmonary circuit undergo damaging changes which eventually become irreversible. The extent of the dilation and hypertrophy will be dependent primarily on the size of the defect but will, also, increase with age. In cases such as that illustrated in figure 4 , when the individual lives to advanced years the heart becomes of enormous size, justifying the older pathologists designation of it as a "cor bovinum."

With the characteristic postnatal conditions as a background let us turn now to the underlying embryological conditions involved in the persistence of interatrial foramen primum. The general story of the origin of septum primum as a crescentic fold of endothelially clothed cardiac muscle arising on the cephalodorsal wall of the atrium has long been well known (Born, 1889; Tandler, '12; Odgers, '35; Patten, '60). In its growth the tips of this crescent-shaped septum are directed toward the endocardial cushions of the atrioventricular canal. The diminishing transatrial communication bounded by the concavity of the septum above and the atrioventricular cushions below, is interatrial foramen primum or more briefly ostium primum (fig. 5). One of the details which seems to have been overlooked is the fact that the free margin of the septum is not composed of cardiac muscle but is a thickened flange of mesenchyme (figs. 5, 6). This is important, for in the closure of ostium primum it is this mesenchymal margin that ultimately fuses 


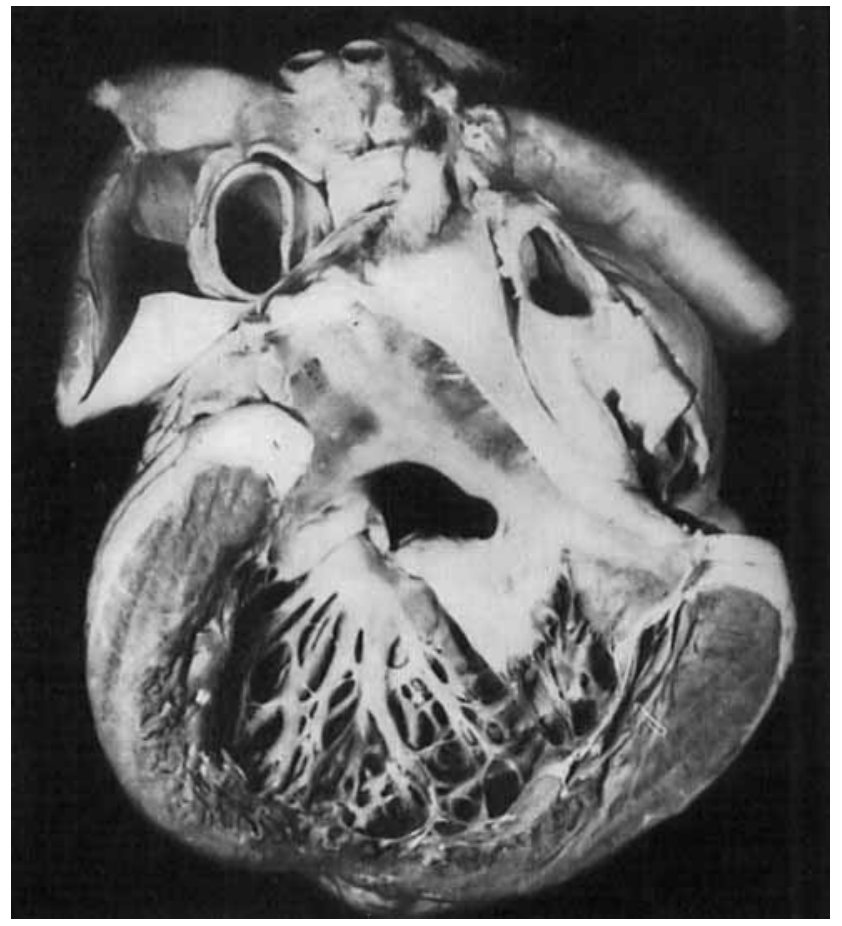

Fig. 2 Persistent interatrial foramen primum near the middle of the usual range of extensiveness. (From heart of 22-year-old female, Path.-anat. Inst., Vienna.)

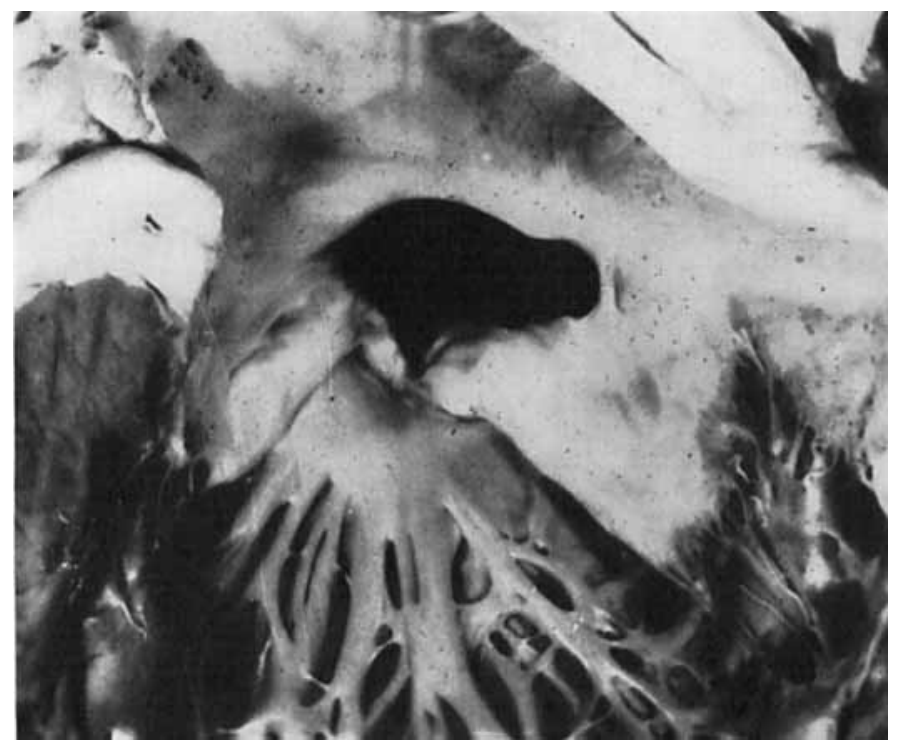

Fig. 3 Detail of cleft in the atrioventricular valves from case illustrated in figure 2. 


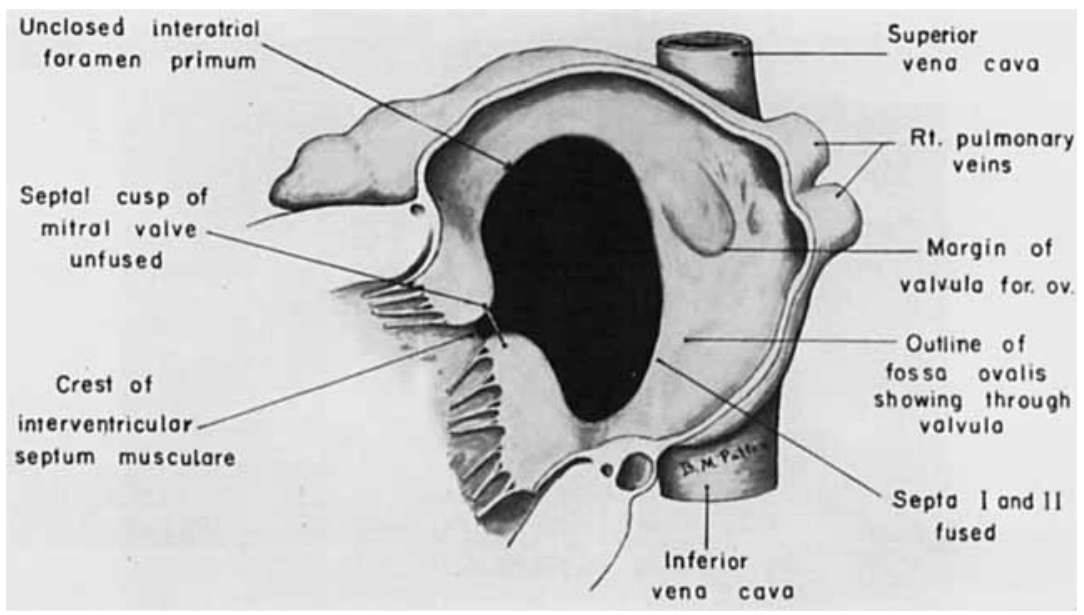

Fig. 4 Persistent interatrial foramen primum of unusually large size. The actual dimensions were $57 \mathrm{~mm}$ in vertical diameter, and $40 \mathrm{~mm}$ across the defect to the bottom of the valve cleft. (Drawn from a specimen in the Rokitanski Museum, Vienna.)

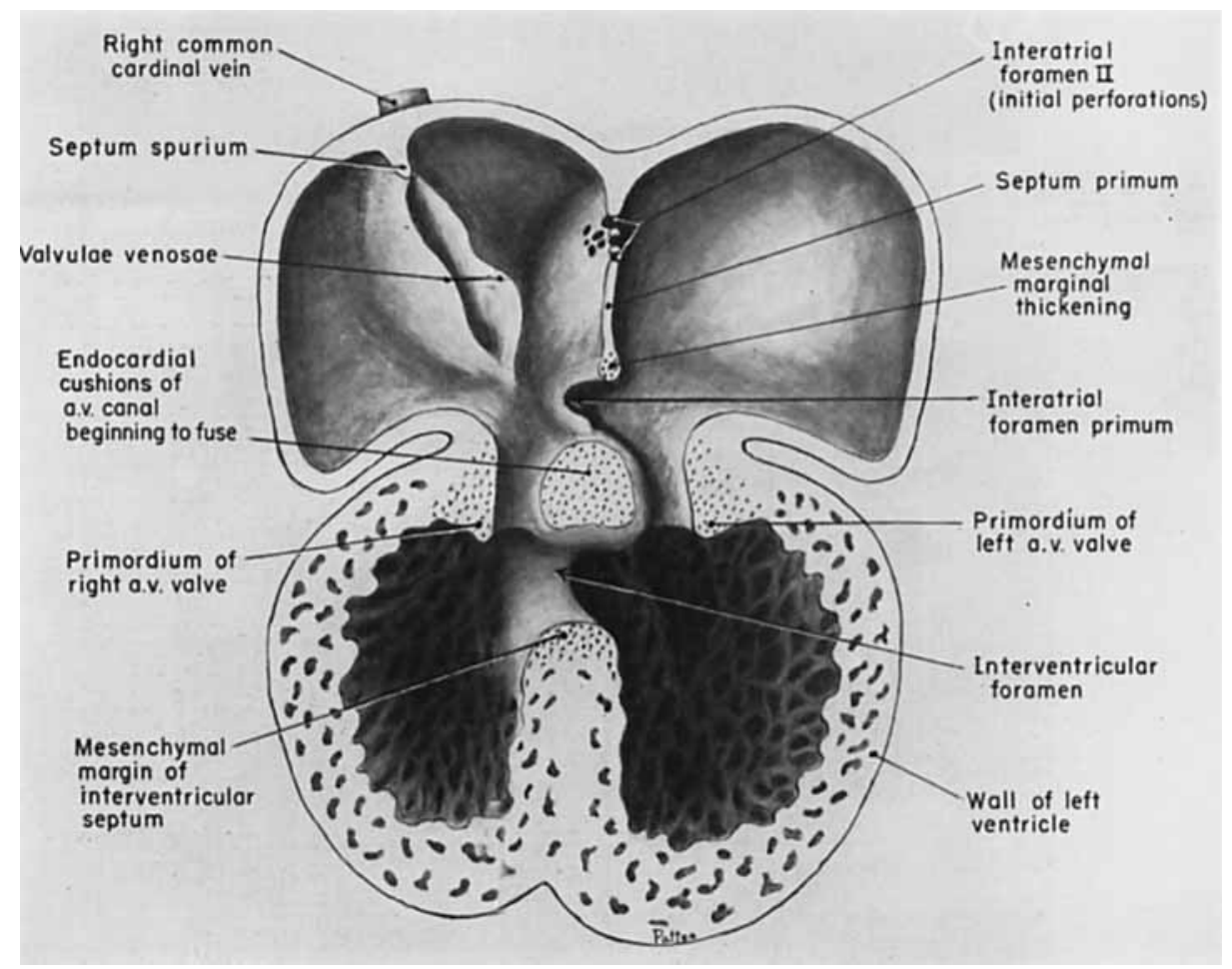

Fig. 5 Semischematic drawing showing the interior of the developing heart in the 6th week at the stage when interatrial foramen primum is almost closed, and interatrial foramen secundum is just being established by the appearance of multiple small perforations. 
with the mesenchymal tissue of the endocardial cushions of the atrioventricular canal (fig. 7).

If one reviews mentally the other places where fusions occur in the normal development of the heart, it is striking that they also involve a similar process with the endothelium promptly losing its continuity at the points of contact and a mesenchyme to mesenchyme fusion being established. Specifically this is what happens in the fusion of the truncoconal ridges which divide the truncus arteriosus into ascending aorta and pulmonary trunk. It occurs also in the fusion of dorsal and ventral endocardial cushions in the division of the atrioventricular canal, and the fusion of the mesenchymal margin of the interventricular septum with atrioventricular canal cushions and conal ridges in the closure of the interventricular foramen. It is my conviction that the consistency with which this sequence of events is repeated in various locations in the developing heart is highly significant from the standpoint of developmental mechanics. Currently the behavior of the endothelial and mesenchymal components in such fusions is being restudied in this laboratory.

Figure 8 suggests by a series of contour lines the successive positions occupied by the margin of septum primum in its growth toward the atrioventricular canal cushions. The numbers are not intended to represent a precise time scale, but merely to serve as useful tags in discussing the progress of events. Subject to some individual variability the start of interatrial septum primum suggested by line number 1 can be recognized in embryos of about 4 weeks (C. R. approximately $4 \mathrm{~mm}$, Streeter's Horizon XIII).

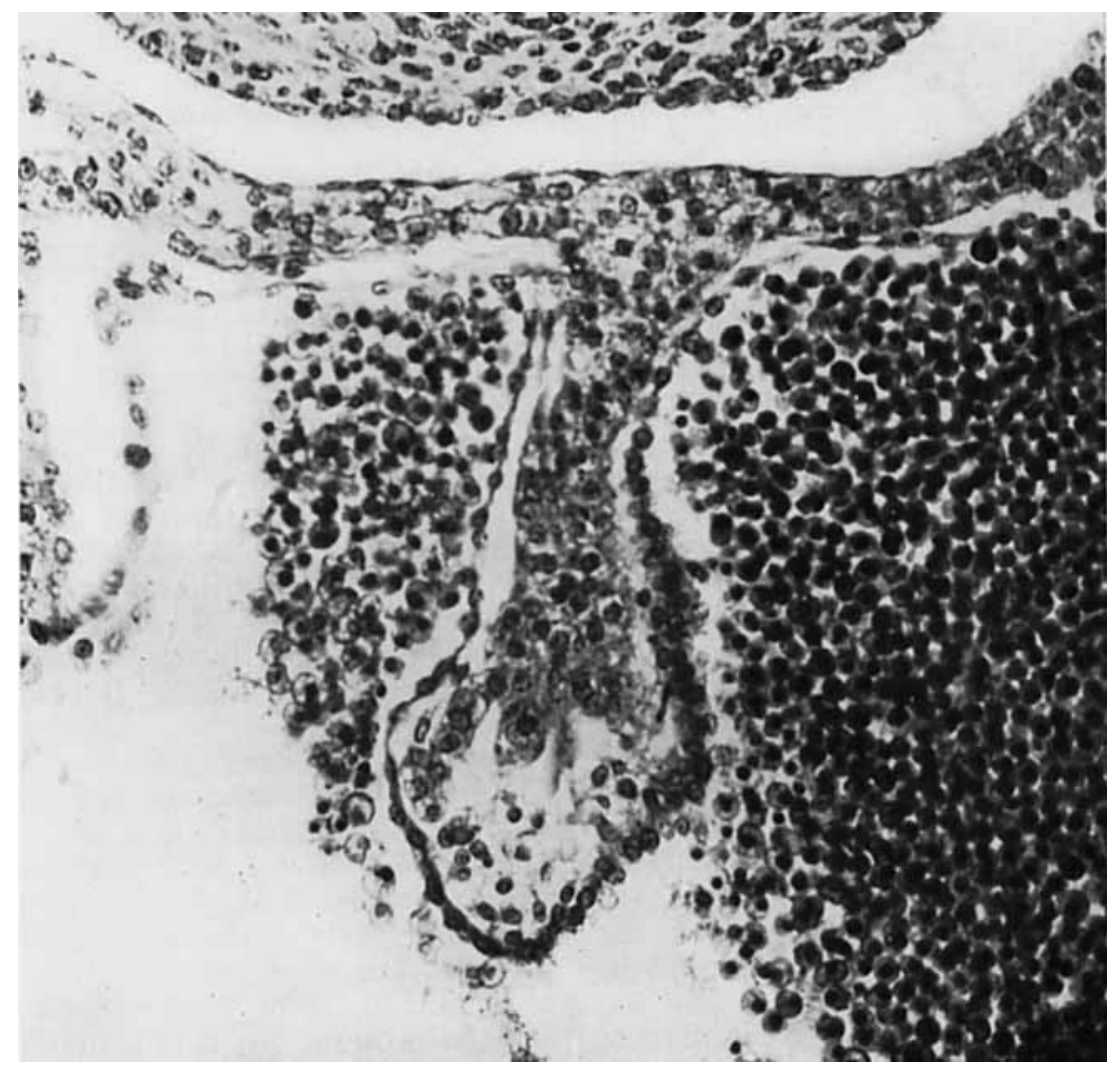

Fig. 6 Photomicrograph. $(\times 260)$ of interatrial septum primum to show its mesenchymal border. (From Univ. of Michigan Collection, E H 396, C. R. $7.5 \mathrm{~mm}$.) 


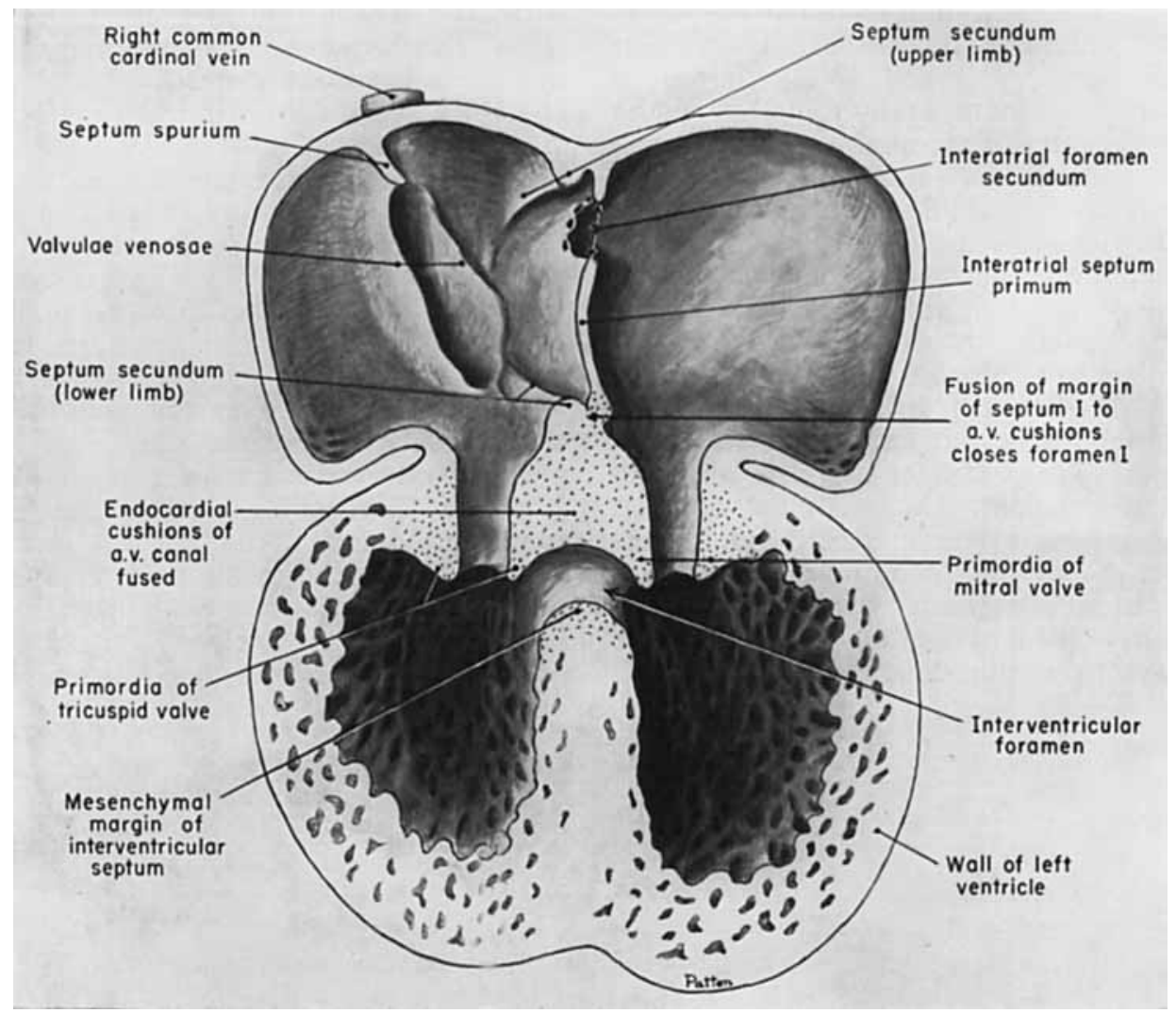

Fig. 7 Semischematic drawing showing the interior of the developing heart at the stage when interatrial foramen primum has been closed and interatrial foramen secundum well opened. Septum secundum is just being established to the right of septum primum.

The atrioventricular canal cushions make contact and begin to fuse during the 6 th week, more specifically in embryos of about $9 \mathrm{~mm}$ (Streeter's Horizon XVI). The fusion of septum primum with the atrioventricular canal cushions to close foramen primum normally occurs in embryos between 10 and $11 \mathrm{~mm}$ (Streeter's Horizon XVII). Thus the numbered lines 2 to 7 suggest the progressive advance of septum primum which occurs between 4 and $10 \mathrm{~mm}$.

Just about the time the atrioventricular cushions begin to fuse, interatrial foramen secundum makes its appearance in the form of a group of minute perforations in the upper part of septum primum (fig. 5). These initial small openings rapidly expand and merge to form a sizable interatrial ostium secundum. It is functionally highly significant that ostium secundum is well established by the time ostium primum closes (fig. 7). Were this not to occur, the left atrium would be cut off with an inadequate blood volume because the return from the lungs is minimal at this stage, and the left side of the heart is dependent on a transeptal shunt from the right for maintaining a balanced intake.

Septum secundum arises to the right of septum primum after ostium secundum has been established and after septum primum has fused with atrioventricular canal cushions (fig. 7). It cannot usually be clearly demonstrated until well into the 7th week of development. It is crescentic in form as was the case with septum primum but it is a much heavier structure and the open end of its crescentic margin is differently directed. Instead of progressing toward the atrioven- 
tricular canal as did septum primum it grows toward the lower part of the sinus opening which ultimately becomes the inferior caval inlet. Figure 9 emphasizes the different direction of growth of septum secundum by superimposing heavy contour lines on light dotted lines indicating the direction of growth of septum primum.
As was the case with septum primum, the contour lines for septum secundum do not purport to be a precise time scale. The number 1 line suggests the location of the margin of septum secundum when it first becomes well established during the 7 th week. The line numbered 6 is at about the location reached by the 6th

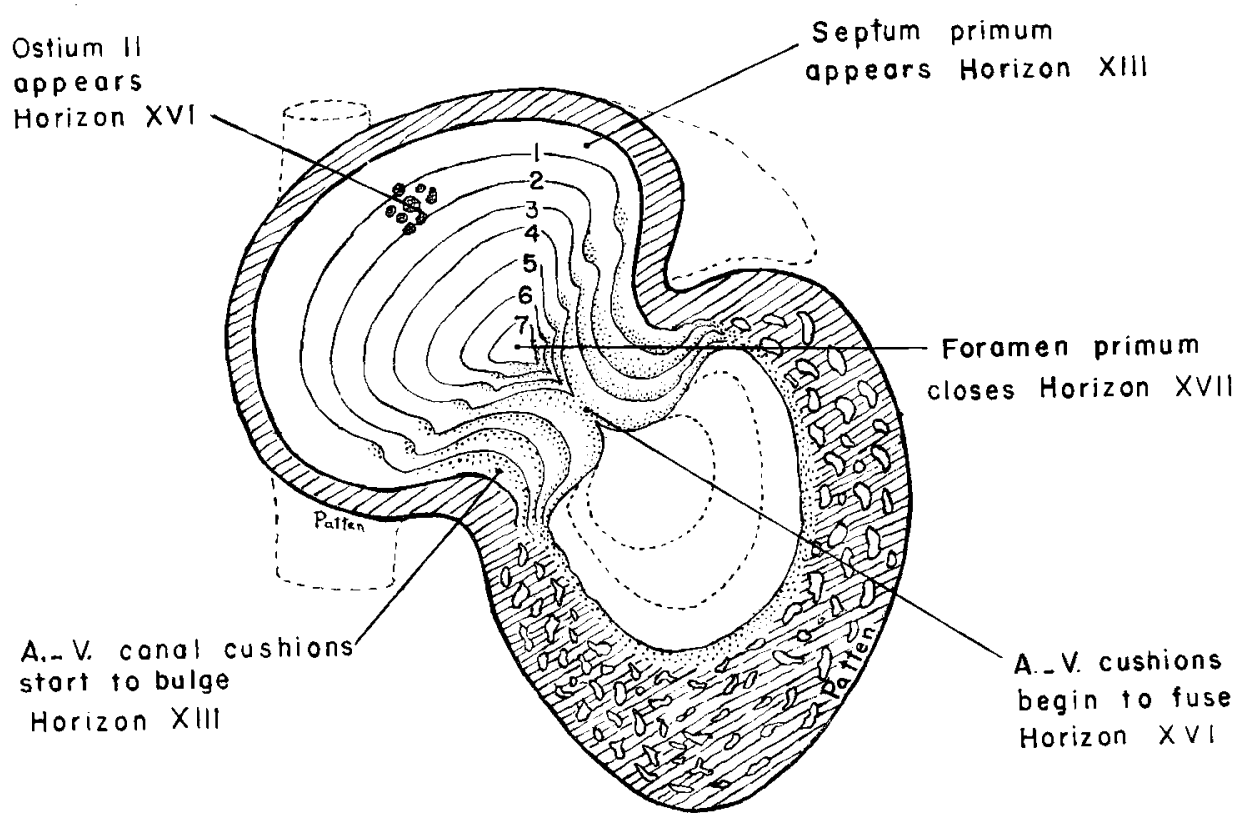

Fig. 8 Schematic diagram showing by a series of contour lines the progress in the growth of interatrial septum primum and of the atrioventricular canal cushions.

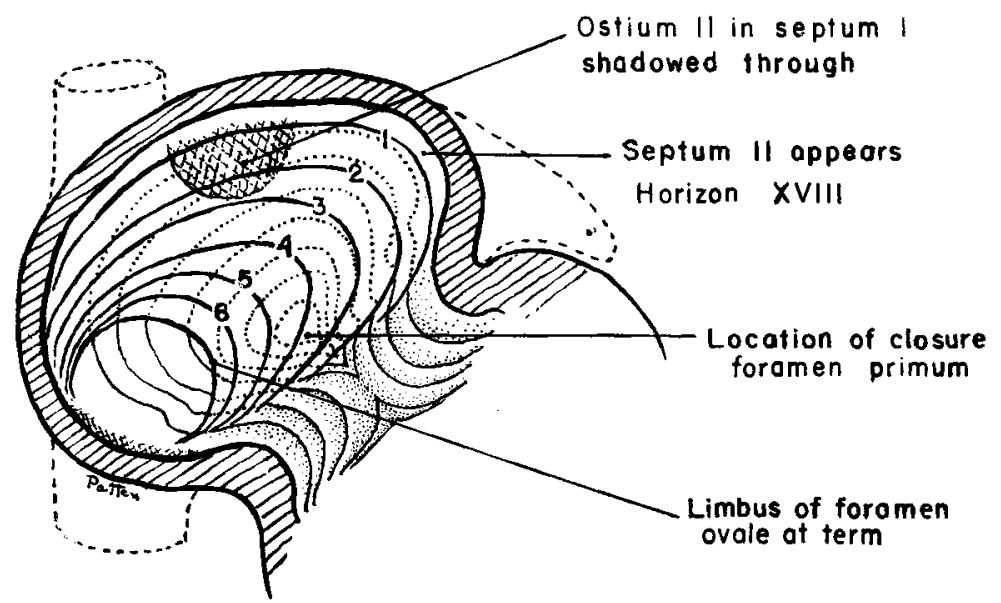

Fig. 9 Schematic diagram showing by a series of contour lines the progress of growth of septum secundum. By dotting in the contour lines for septum primum the difference in the direction of growth of the two septa is emphasized. 
month. It is worth emphasizing that the periods of rapid growth of the two interatrial septa are radically different, that of septum primum being between 4 and 6 weeks and that of septum secundum being from the 7 th week through the 6th month and even extending at a reduced rate into the final trimester of pregnancy.

From the standpoint of a persisting ostium primum this difference in timing is highly significant. It means that in the normal development of the interatrial septal complex, septum secundum follows along the right side of an already completed septum primum and merges with it to form the thick lower portion of the septum (see especially contour lines 2-4 in fig. 9). When septum primum fails to close ostium primum, septum secundum, deprived of its support as a sort of "scaffolding," fails to grow into the region of the defect. The way its growth seems to be distorted is indicated schematically by the contour lines in figure 10 . Thus from the developmental standpoint the crucial departure from normal in a persistent ostium primum is deficient growth of septum primum between 4 and 6 weeks. This in turn means that if environmental factors were involved they must have been operative not later than this period and in all probability early in it, or even a little before the active growth was due to commence.
It is of interest also that a disturbance starting at a very early period in one structure has such a striking effect on the course of development at a much later stage of development in another related structure. With this course of events in mind we can properly speak of a persisting interatrial foramen primum but we should not refer to the condition as "a defect of septum primum" for septum secundum, also, is definitely involved.

Another significant thing is the varying extent to which the atrioventricular canal cushions may be involved with a persistent ostium primum. In the cases illustrated in figures 2 and 4 it is quite evident from the notched atrioventricular valves that the canal cushions have failed to merge completely. In such cases it seems not unlikely that defective growth of the cushions may be the real primary factor. The advancing tips of crescentic septum primum lie on the atrial faces of the canal cushions (fig. 5) and may well follow their lead in closing or failing to close foramen primum.

In contrast to the foregoing more usual conditions, are the exceptional cases such as that illustrated in figure 11. Here the completeness of the base of the atrioventricular valves indicates that the growth and fusion of the canal cushions must have been essentially normal. There is, to be sure, a slight notch on the free

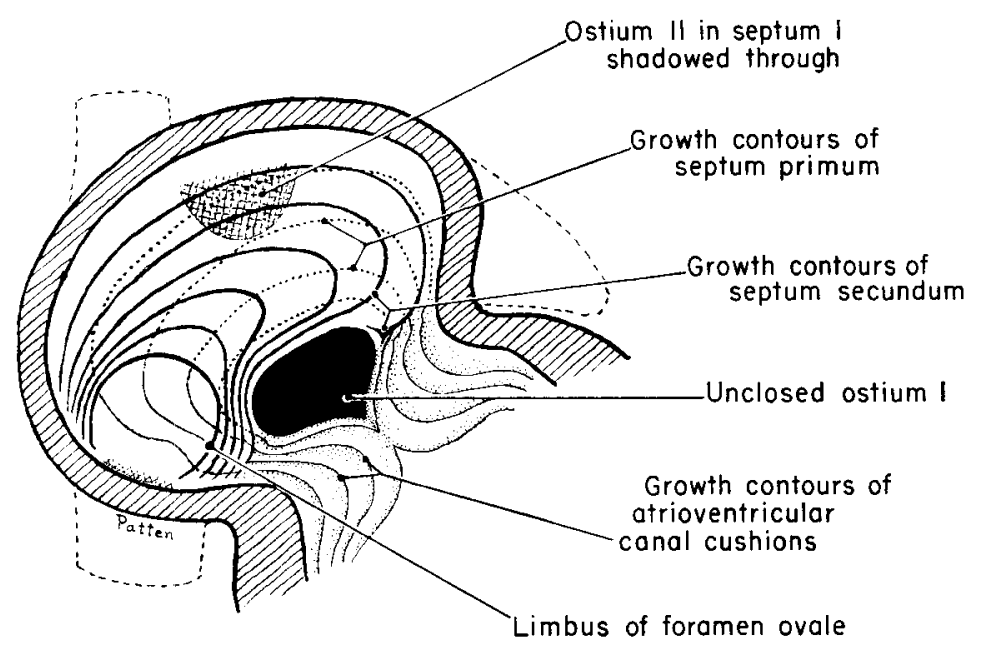

Fig. 10 Schematic diagram showing by heavy contour lines the way the growth of septum secundum is modified in the presence of a persisting interatrial foramen primum. 
margin of the valve leaflet but that appears to be of little more significance than other similar variations often seen in the configuration of the valves. It seems evident, therefore, that in cases of this type the defect is due to inadequate growth of septum primum with no involvement of the atrioventricular canal cushions.
At the other extreme is the case illustrated in figure 12. Here there can be no question about the markedly defective growth of the atrioventricular canal cushions. The abnormality is really a combination of persistent ostium primum, an undivided atrioventricular canal, and absence of the membranous portion of the inter-

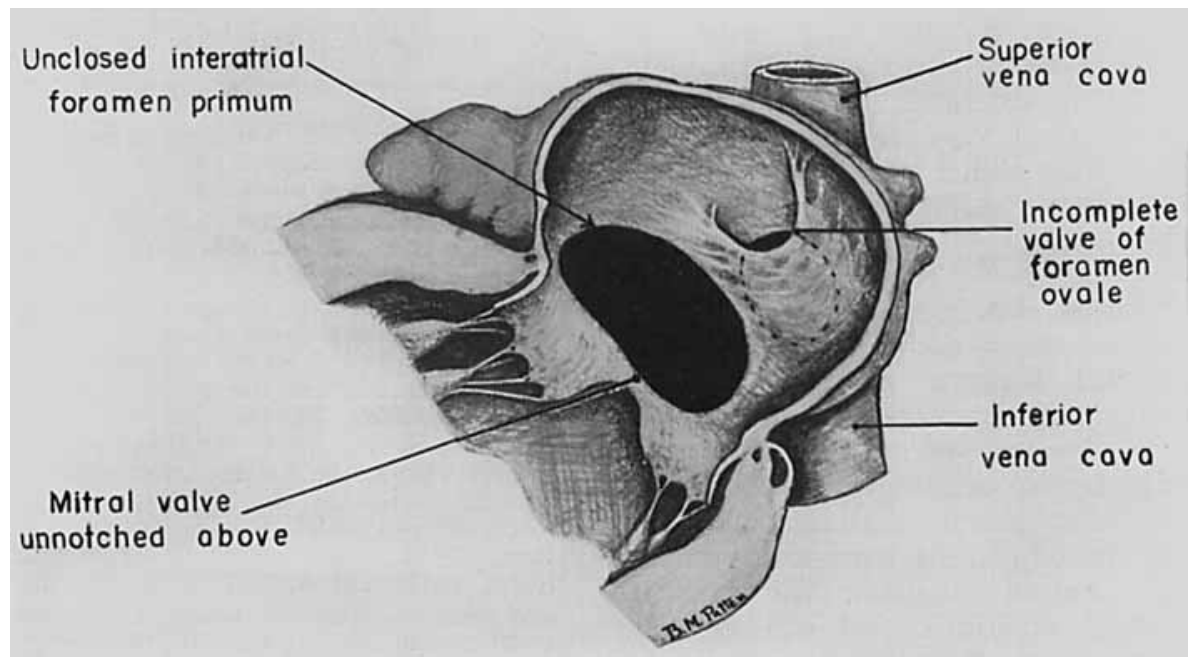

Fig. 11 Case of interatrial foramen primum in which the atrioventricular valves were unnotched at their base. (From the Rokitanski Museum, Vienna. This specimen from a 19-year-old male was described by Rokitanski (1875) as his case no, 4, but not illustrated.)

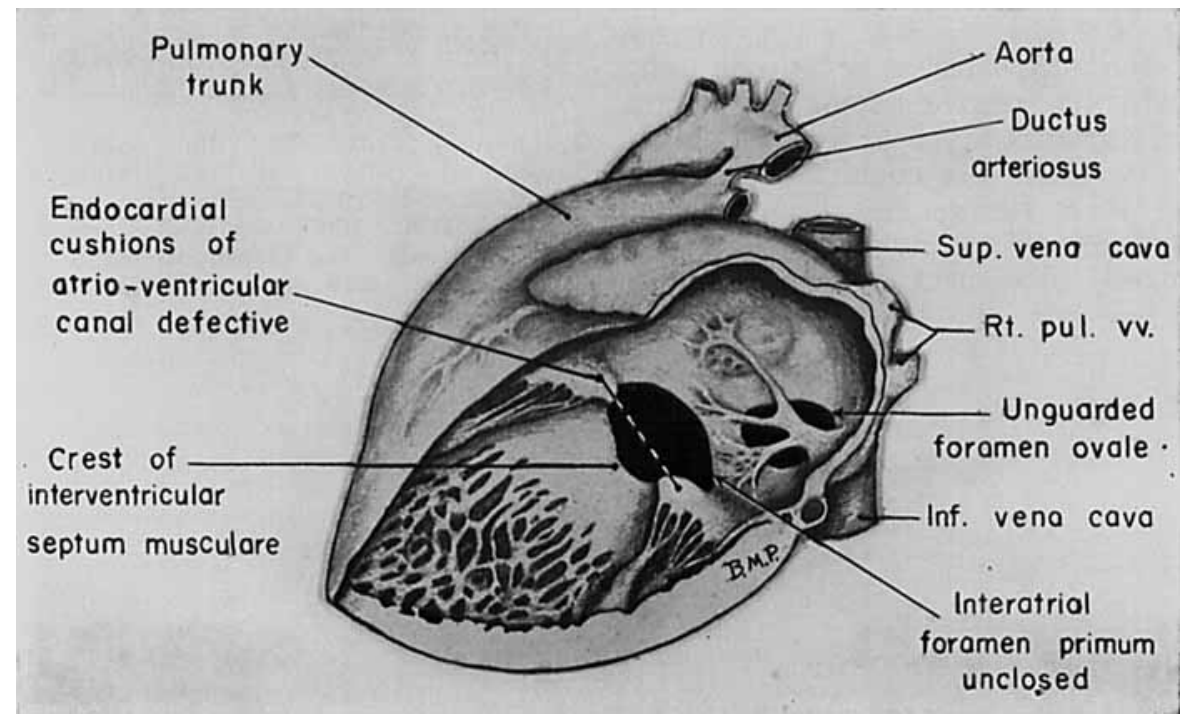

Fig. 12 Persistent interatrial foramen primum combined with markedly deficient growth of the atrioventricular canal cushions. (Heart of child, no clinical record except "Polizeilich secirt," Rokitanski Museum, Vienna.) 
ventricular septum. A comparison of this case with that illustrated in figures 2 and 3 , however, should make it apparent that we are dealing with the same fundamental developmental distortions. In both cases the growth of the interatrial septal complex and the fusion of the atrioventricular canal cushions have been defcient. The differences lie merely in the relative extent to which the two processes have been involved. Any large series of cases of persistent interatrial foramen primum can be expected to show a complete spectrum of variations in the relative extent with which these closely associated processes exhibit defective growth.

\section{SUMMARY}

A persistent interatrial foramen primum always involves defective growth of interatrial septum primum and a secondarily associated disturbance in the growth of septum secundum. In most cases there is also deficient growth of the atrioventricular canal cushions as evidenced by the gap in the base of the atrioventricular valves. Because the growth of the canal cushions and the growth of septum primum normally progress concurrently, it is difficult to be certain in many instances which has been the primary causative factor in an unclosed ostium primum. The fact that in the majority of cases there is a gap in the base of the atrioventricular valves indicates that the atrioventricular cushions are more regularly involved than has been recognized. Since the cushions normally complete their fusion just prior to the closure of ostium primum it seems not unreasonable to suspect that in many in- stances their deficient growth may be the true primary factor in a failure to close ostium primum. The more marked the gap in the base of the atrioventricular valves the more deeply the cushions would appear to be implicated. It should be borne in mind, however, that the rare cases without a gap in the valves clearly show that it is possible for a persistent ostium primum to be the result of defective septal growth alone.

\section{LITERATURE CITED}

Abbott, M. E. 1932 Congenital heart disease. Part II. Causes and types of heart disease. Nelson Looseleaf Medicine, 4: 207-321.

Born, G. 1889 Beiträge zur Entwicklungsgeschichte des Saugethierherzens. Arch. Mikr. Anat., 33: 284-377.

Edwards, J. E. 1960 Congenital malformations of the heart and great vessels. A. Malformations of the atrial septal complex. In: Gould, S. E., Pathology of the Heart, 2nd ed. C. C Thomas, Chap. VI, pp. 260-293.

Odgers, P. N. B. 1935 The formation of the venous valves, the foramen secundum and the septum secundum in the human heart. J. Anat., 69: 412-422.

Patten, B. M. 1960 The development of the heart. In: Gould, S. E., Pathology of the Heart, 2nd ed. C. C Thomas, chap. II, pp. 24-92.

Peacock, T. B. 1866 On Malformations of the Human Heart, 2nd ed., John Churchill and Sons, London, $\mathrm{XV}+204 \mathrm{pp}$.

Rogers, H. M., and J. E. Edwards 1948 Incomplete division of atrioventricular canal with patent interatrial foramen primum (persistent common atrioventricular ostium). Am. Heart J., 36: 28-54.

Rokitansky, C. F. (von) 1875 Die Defecte der Scheidewände des Herzen. Braumüller, Wien, p. 157.

Tandler, J. 1912 The Development of the Heart. In: Keibel and Mall, Human Embryology, 2: 534-570.

Taussig, H. B. 1947 Congenital Malformations of the Heart. The Commonwealth Fund, New York, $x \times x i+618$ pp. 